\title{
On Some Gauss and Lobatto Based Integration Formulae
}

\author{
By T. N. L. Patterson
}

1. Introduction. The economy of the Gaussian quadrature formulae for carrying out numerical integration is to some extent reduced by the fact that an increase in the order of the formulae makes no use of previous integrand evaluations. Kronrod [1] has shown how the Gauss formula of degree $2 n-1$ can be extended to one of degree $3 n+2$ by making use of the original $n$ Gauss points and an additional set of $n$ points. However, it is not possible to proceed further than this without using an entirely new set of points with a resulting waste of computational labor. It may be noted that due to the absence of a convenient error estimate for the Gaussian formulae it is usually necessary to carry out a quadrature using more than one order of formulae to check the convergence.

In this paper a set of integration formulae is derived based on a set of $2^{r}+1$ Gauss or Lobatto points, where $r$ is an integer. If the original points are denoted by $x_{j}, j=1,2, \cdots,\left(2^{r}+1\right)$, then $r$ subsets of points $x_{2 i(j-1)+1}, j=1,2, \cdots$, $\left(2^{r-i}+1\right)$ are obtained for $i=1,2, \cdots, r$ by successively deleting alternate points from the preceding subset. The integration weights associated with each subset can be determined as described in Section 2. In carrying out an integration the number of points is successively increased until convergence appears satisfactory or until the number of points reaches $2^{r}+1$ corresponding to the full accuracy of the Gauss or Lobatto formulae. Since each subset includes the points of the previous subset, no integrand evaluations are wasted. The degree of precision of the successive formulae are one less than the number of points used, since they are of the Newton-Cotes type. For the base formula, the Newton-Cotes weights and the base weights degenerate of course to one and the same.

The integration formulae have been derived for the basic set of 33 Gauss points $(r=5)$ as well as for the sets of 65 Gauss and Lobatto points $(r=6)$. It was considered that the 65-point Gauss and Lobatto formulae were capable of dealing with all but pathological integrands so that it was unnecessary to base the formulae on higher values of $r$. For example, the Gauss 65-point formula will integrate powers of $x$ up to 154 with a relative accuracy of just less than nine decimal digits.

The Chebyshev expansion method of Clenshaw and Curtiss [2] has also the characteristic of not wasting previous integrand evaluations, and a comparison with the new formulae is therefore presented in Section 3. The Clenshaw-Curtiss quadrature formulae may be interpreted as the Newton-Cotes formulae with the abscissas $x_{k}=\cos (k \pi /(n-1)), \quad k=0,1, \cdots, n-1$. These formulae will be referred to later as Chebyshev formulae.

2. Evaluation of the Integration Weights. The Lagrangian interpolating poly-

Received August 7, 1967. Revised January 15, 1968. 
nomial of degree $n-1$ for a function $f(x)$ given at the points $x_{i}, i=1, \cdots, n$, is

$$
P(x)=\sum_{i=1}^{n} L_{i}(x) f\left(x_{i}\right)
$$

where

$$
L_{i}(x)=F_{i}(x) / F_{i}\left(x_{i}\right)
$$

and

$$
F_{i}(x)=\prod_{j=1}^{k}\left(x-x_{j}\right) /\left(x-x_{i}\right) .
$$

Thus the weights of an $n$-point integration formula

$$
\int_{-1}^{1} f(x) d x=\sum_{i=1}^{n} \omega_{i} f\left(x_{i}\right)
$$

are given by

$$
\omega_{i}=\int_{-1}^{1} L_{i}(x) d x
$$

These weights can be evaluated exactly in a numerically stable fashion using a Gauss formula with $n / 2$ points when $n$ is even and $(n+1) / 2$ points when $n$ is odd. It is to be noted that the weights for any quadrature formula, including the Gaussian and Lobatto formulae, can be calculated in this way.

If the derivatives of the integral are known as well, the Hermite interpolation formula allow the weights $B_{i}$ and $C_{i}$ of the formula

$$
\int_{-1}^{1} f(x) d x=\sum_{i=1}^{n} B_{i} f\left(x_{i}\right)+\sum_{i=1}^{n} C_{i} f^{\prime}\left(x_{i}\right)
$$

to be obtained from

$$
\begin{aligned}
& B_{i}=\int_{-1}^{1}\left[1-2 L_{i}{ }^{\prime}\left(x_{i}\right)\left(x-x_{i}\right)\right] L_{i}{ }^{2}(x) d x \\
& C_{i}=\int_{-1}^{1}\left(x-x_{i}\right) L_{i}{ }^{2}(x) d x .
\end{aligned}
$$

These weights can be evaluated exactly using a Gauss formula with $n$ points. As Lanczos [3] has noted, the inclusion of the $n$ values of the derivatives in an arbitrary point formula gives rise to an integration formula with the same integrating power as the $n$-point Gauss formula.

3. New Formulae. By the method of Section 2 integration formulae with 5,9 , and 17 points were derived, based on the 33 Gauss points, together with formulae with 5, 9, 17 and 33 points based on the 65 Gauss and Lobatto points. The Chebyshev formulae for $5,9,17,33$ and 65 , were also derived for comparison. The integrand weights for the Lobatto, Gauss and Chebyshev formulae are given in Tables* M1, M2, M3 and M4. All the Gauss formulae used to evaluate the weights were obtained from Gawlik [4]. The weights associated with the 65-point Lobatto formula have been given by Rabinowitz [5] and have not been included in Table

* The letter $M$ preceding a table number refers to the microfiche card. 
M1. The 65 Gauss points and weights have not previously been tabulated and had to be calculated to derive Table M2. To assess the integrating power of the formulae each was applied to integrate powers of $x$ higher than those which should be integrated exactly. In Figs. 1 to 4 the modulus of the fractional error (defined as the ratio of the error to the true value of the integral) committed by the various formulae is plotted against the power of $x$ being integrated. It can be seen that the formulae based on the 33 Gauss points probably give the best overall performance. The Lobatto formulae appear to be particularly good at integrating very high powers of $x$. A further indication of this is evident from Table 1, which records the performance of the various formulae when integrating $\int_{-1}^{1}\left|x+\frac{1}{2}\right|^{1 / 2} d x$, whose integrand has a singularity in its derivatives at $x=-\frac{1}{2}$. This integrand could

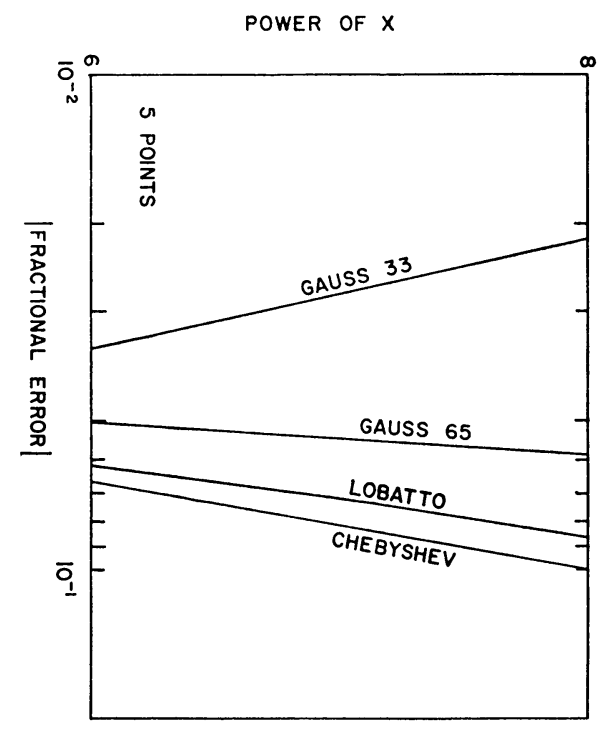

Figure 1

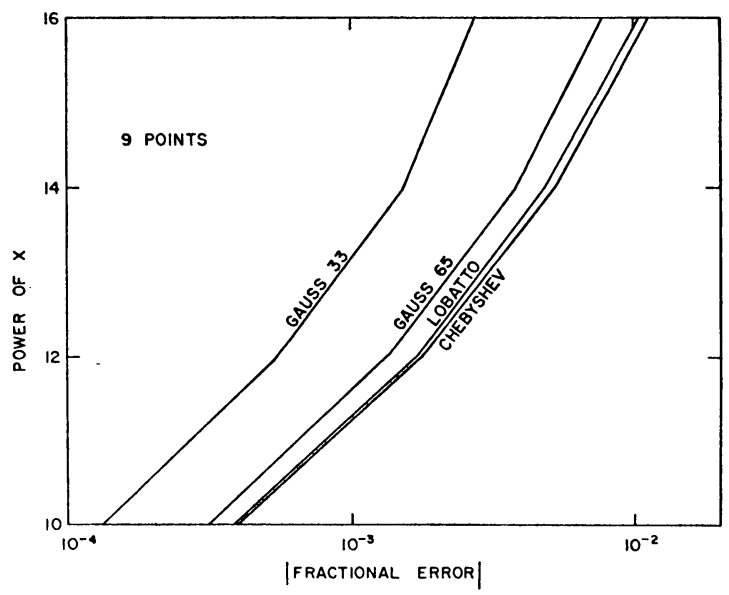

Figure 2 


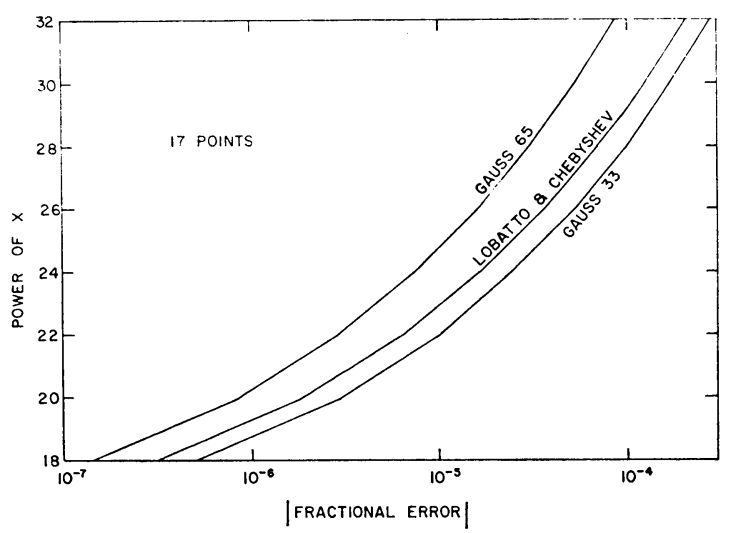

Figure 3

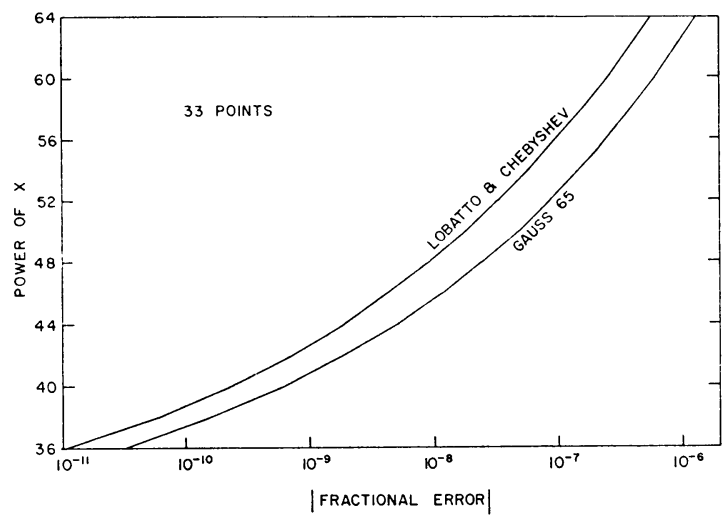

Figure 4

TABLE 1

Errors in Calculating $\int_{-1}^{1}|x+1 / 2|^{1 / 2} d x$

\begin{tabular}{l|ccccc} 
Basic points & \multicolumn{5}{|c}{ Number of points } \\
\cline { 2 - 6 } & 5 & 9 & 17 & 33 & 65 \\
Gauss 65 & 0.0569 & 0.0180 & 0.0041 & 0.0029 & -0.0011 \\
Gauss 33 & 0.0507 & 0.0194 & 0.0011 & 0.0026 & $-\overline{0.00078}$ \\
Chebyshev & 0.0627 & 0.0160 & 0.0064 & 0.0021 & -0.00039 \\
Lobatto 65 & 0.0608 & 0.0168 & 0.0058 & 0.0025 & 0.0039 \\
\hline
\end{tabular}

probably only be approximated with acceptable accuracy by a polynomial of very high degree. It would thus seem that the Lobatto based formulae may provide a useful scheme for controlling the accuracy and economy of numerical integrations.

The tables were calculated using at least thirty-digit arithmetic and are correct to all figures given. The usual checks of integration of powers were successfully applied. It is interesting to note that the weights of all the formulae are positive so that their stability is likely to be high. 
Acknowledgement. This work was commenced at the Queen's University of Belfast and completed at the Southwest Center for Advanced Studies, Dallas, Texas. It was sponsored in part by the Air Force Cambridge Research Laboratories, through the European Office of Aerospace Research, OAR, United States Air Force, under contract No. AF61(052)-780 and in part by the National Aeronautics and Space Administration Grant NsG-269.

Southwest Center for Advanced Studies

Dallas, Texas 75230

1. A. S. Kronnon, Nodes and Weights of Quadrature Formulas, English transl. from Russian, Consultants Bureau, New York, 1965. MR 32 \#597.

2. C. W. Clenshaw \& A. R. Curtiss, "A method for numerical integration on an automatic computer," Numer. Math., v. 2, 1960, pp. 197-205. MR $22 \# 8659$.

3. C. Lanczos, Applied Analysis, Prentice-Hall, Englewood Cliffs, N. J., 1956. MR 18, 823.

4. H. J. Gawlik, Zeros of Legendre Polynomials of Orders $2-64$ and Weight Coefficients of Gauss Quadrature Formulae, Armament Research and Development Establishment Memorandum (B) $77 / 58$, Fort Halstead, Kent, England, 1958.

5. P. Rabinowirz, "Abscissas and weights for Lobatto quadrature of high order," Math. Comp., v. 14, 1960, pp. 47-52. MR $22 \# 1076$. 